\title{
Spectral Shifting in Extraordinary Optical Transmission by Polarization-Dependent Surface Plasmon Coupling
}

\author{
Jongkyoon Park ${ }^{1} \cdot$ Hyunsoo Lee ${ }^{1} \cdot$ Alexander Gliserin ${ }^{1} \cdot$ Kyujung Kim $^{1,2} \cdot$ Seungchul Kim ${ }^{1,2}$ (D)
}

Received: 28 July 2019 / Accepted: 11 October 2019 / Published online: 16 November 2019

(C) The Author(s) 2019

\begin{abstract}
Nanoapertures in a metallic film exhibit extraordinary optical transmission (EOT) owing to the surface plasmon resonance. Their transmission properties are known to be dependent on the structural parameters of the nanoapertures. In addition, the polarization of light has also a crucial influence on the transmission spectrum. In this study, we numerically found that the polarization state is a sensitive parameter in plasmonic EOT only when the gap size between triangular nanoapertures is less than $\sim 20 \mathrm{~nm}$. For a polarization of the light perpendicular to the axis between the nanoapertures, the optical transmission spectrum is nonlinearly redshifted with decreasing gap size. This spectral shifting of the transmission has potential applications for active optical filters, which can be manipulated by the polarization of light or by adjusting the gap size.
\end{abstract}

Keywords Extraordinary optical transmission $\cdot$ Surface plasmon $\cdot$ Nanoplasmonics

\section{Introduction}

In 1998, T.W. Ebbesen et al. firstly reported that nanoscale hole apertures in a metallic film induce extraordinary optical transmission (EOT), in which high transmission occurs at the resonance wavelength [1]. The origin of EOT is mainly known to be the surface plasmons (SP), which are collective charge oscillations due to the resonant interaction between light and free electrons at a metal-dielectric interface [2-4]. A typical EOT structure consists of a periodic nanoaperture array and its optical properties such as transmission spectrum, efficiency, and linewidth depend on the complex permittivity of the materials and design parameters (hole size, pitch, and aperture shape) [5-7]. Since a SP can only be efficiently induced in designated nanoapertures or shaped nanostructures [8], the SP enables sensitive bio-sensing [9], sub-wavelength scale beam focusing or lithography $[10,11]$, novel optical

Jongkyoon Park and Hyunsoo Lee contributed equally to this work.

Seungchul Kim

s.kim@pusan.ac.kr

1 Department of Cogno-Mechatronics Engineering, College of Nanoscience and Nanotechnology, Pusan National University, Busan 46241, South Korea

2 Department of Optics and Mechatronics Engineering, College of Nanoscience and Nanotechnology, Pusan National University, Busan 46241, South Korea filtering [12], plasmonic metrology [13], terahertz imaging [14], and next-generation photonic devices [15-18]. Recently, electronically tunable EOT experiments in graphene plasmonic ribbons were introduced, showing that a graphenecoupled sub-wavelength Au slit array exhibits an active EOT structure at mid-infrared frequencies [19].

Since EOT provides selective transmission and efficient focusing of light at the sub-wavelength scale [20], it enables new applications for precise optical filters at the nanoscale. The optical transmission spectrum is tunable by adjusting the pitch of the nanohole array because efficient excitation of SPs primarily depends on the distance between the nanoholes. However, controlling the pitch size of a nanohole array for a significant spectral shift of the optical transmission is difficult in noble metal-based plasmonic structures because conventionally used metal film on a dielectric substrate is not highly stretchable to provide the enough pitch variation.

In this study, we demonstrate that the transmitted EOT spectrum through an asymmetric bow-tie nanoarray can be efficiently tuned by more than $90 \mathrm{~nm}$ in wavelength at the near-infrared regime by changing the gap size between the triangles. This shift of the transmitted optical spectrum only occurs for transverse electric (TE) polarization with respect to the bow-tie axis, whereas light with transverse magnetic (TM) polarization is insensitive to the variation of the bow-tie gap. In particular, the EOT resonance peak shifts nonlinearly by an average of $3.12 \mathrm{~nm}$ per one-nanometer increment of the bowtie gap for the TE polarization when the gap size is less than 20 
nm. Therefore, this EOT-based asymmetric nanoarray can facilitate wavelength-tunable optical filters without mechanical deformation in the field of novel flat optics.

Figure 1a shows the schematic of the polarizationdependent EOT structure based on the nanogap bow-tie. Both the TE and TM polarized light enable the SPs generation on the bow-tie nanoarray, which result in optical transmission to the other side of the metal film. When the TM polarized light is induced on the nanoarray, SPs are generated at the bottom side of each triangular aperture. These SPs propagate along the gold surface but do not reach the gap of bow-tie aperture, which implies no dependence on the gap size for this polarization. On the other hand, SPs are predominantly generated around the nanogap by the TE polarized light, thus, the plasmonic fields are highly confined at the vertex of each triangular aperture, which is closest to the nanogap. When the gap size is reduced to a few tens of nanometers, these two plasmonic fields start to couple, and the resulting resonance condition is significantly affected by the gap size. Because the EOT effect is mainly based on SP resonances, the transmitted spectrum is tunable by changing the bow-tie gap size only if the TE polarized light is used.

\section{Numerical Simulations and Results}

The polarization state of light can significantly affect the SP resonance condition, especially in asymmetric metallic nanoapertures. In order to analyze this phenomenon, we performed finite difference time domain (FDTD) simulations with a unit cell of the bow-tie nanostructure shown in Fig. 1b. For simplicity, we chose fixed geometrical parameters except the gap size. The fixed geometrical parameters in this calculation are a pitch of $600 \mathrm{~nm}$ $\times 300 \mathrm{~nm}$, a thickness of $50 \mathrm{~nm}$, as well as a $90 \mathrm{~nm}$ height, and $60^{\circ}$ internal angles of the triangles. A commercial FDTD software (FDTD Solution, Lumerical, Inc.) was used for all simulations. These parameters were chosen for efficient SP excitation considering typical nanofabrication constraints.

The bow-tie structure in the simulations consists of a free-standing gold film, and its optical properties such as the frequency-dependent complex permittivity were taken from Yakubovsky et al. [21]. Fine adaptive meshes with a step size of $0.5-3.5 \mathrm{~nm}$ were additionally applied at the gap of the bow-tie aperture, while meshes with a step size

(a)

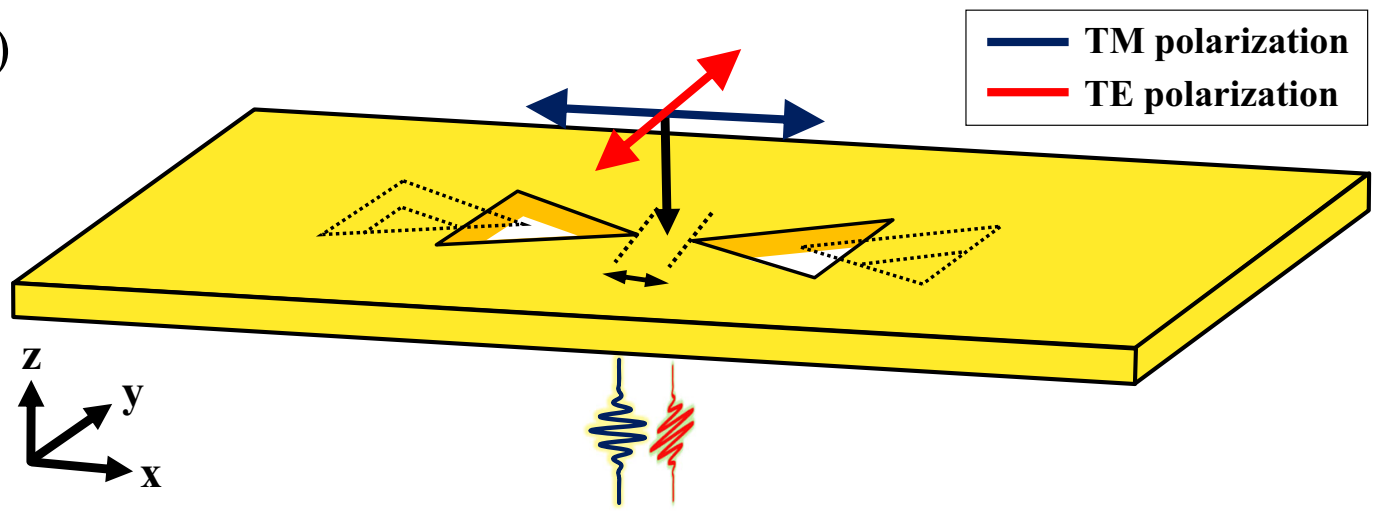

(b)

Top view

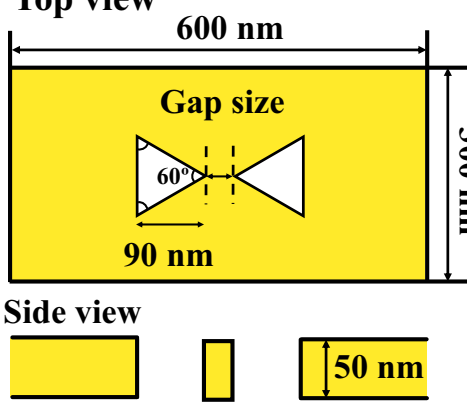

(c)

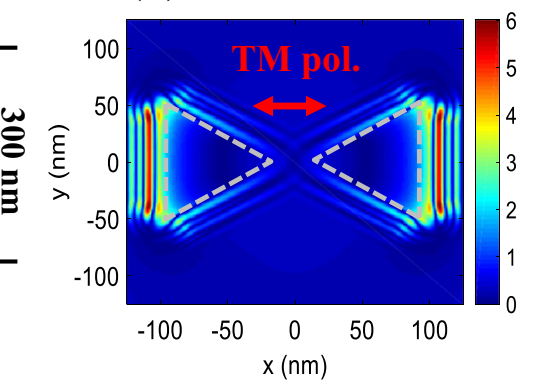

(d)

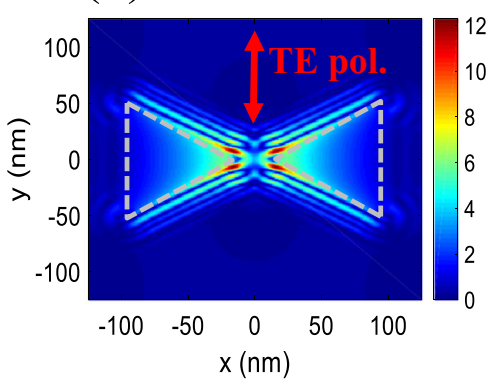

Fig. 1 Scheme of polarization-dependent EOT. a When TM or TE polarized light illuminates a bow-tie nanoarray under normal incidence, surface plasmons are generated, leading to EOT. b Structural parameters of the bow-tie nanoarray. The calculated E-field distribution at the surface of the gold film is shown for $\mathbf{c}$ TM and $\mathbf{d}$ TE polarized light. Dashed lines represent the geometry of the nanoaperture. The E-field is significantly enhanced at the base of each triangular aperture for TM polarized light and at the vertex of each triangle around the nanogap for TE polarized light 
of $1-8 \mathrm{~nm}$ were applied to the entire simulation volume. In addition, an adaptive mesh and a perfectly matched layer (PML) boundary condition were used for the z-axis (out-of-plane) direction, while symmetric and antisymmetry boundary conditions were chosen in-plane for computational simplicity. The bow-tie structure was illuminated by a plane wave with a spectral bandwidth of 450 $1000 \mathrm{~nm}$ in wavelength and a field strength of $1 \mathrm{~V} / \mathrm{m}$, and the transmitted spectrum was monitored for a duration of $100 \mathrm{fs}$ with a step size of 0.93 as.

Figure $2 \mathrm{a}$ and $\mathrm{b}$ show the calculated transmission spectrum as a function of gap size from 0 to $100 \mathrm{~nm}$ for the TM and TE polarization states, respectively. Figures $2 \mathrm{c}$ and d depict representative optical transmission spectra for gap sizes of 0,25 , 50,75 , and $100 \mathrm{~nm}$ for the TM and TE polarization states, respectively. Both polarization states exhibit two distinct resonance peaks at around $500 \mathrm{~nm}$ and $650 \mathrm{~nm}$ wavelength. The resonance peak at $500 \mathrm{~nm}$ does not change with the variation of the gap size in both cases. However, the other peak at approximately $650 \mathrm{~nm}$ slightly redshifts for TM polarization with decreasing gap size. This redshift is significantly more

(a)

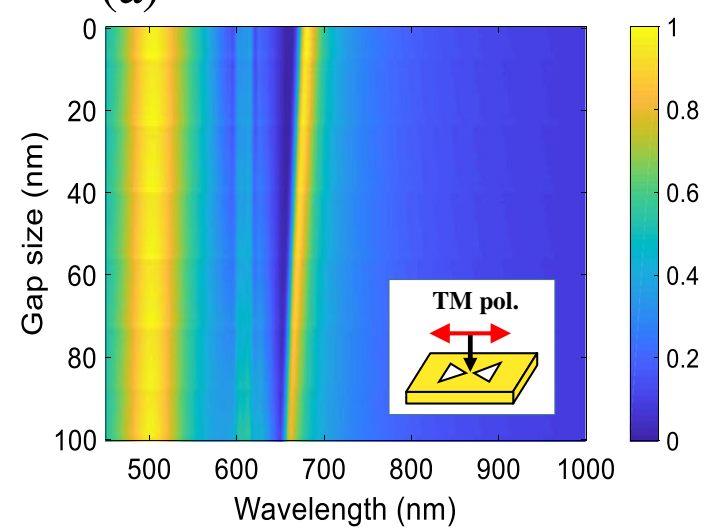

(c)

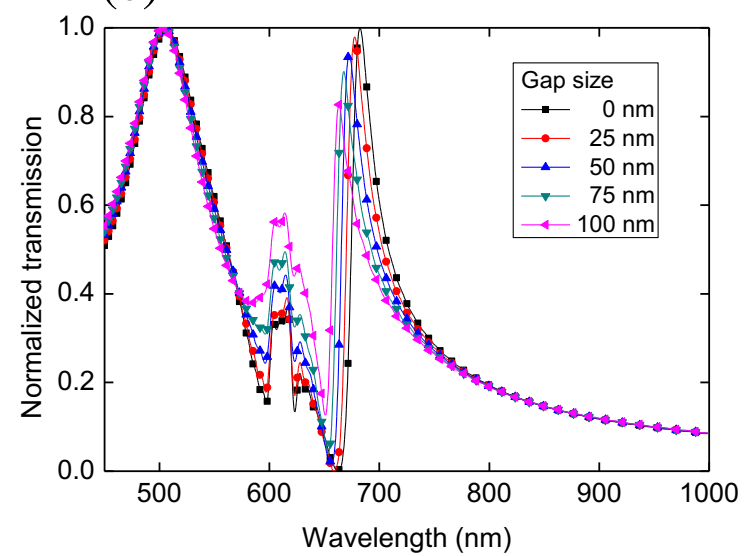

Fig. 2 Calculated transmission spectra for $\mathbf{a}$ TM and $\mathbf{b}$ TE polarized light as a function of gap size. Several representative transmission spectra for different gap sizes are shown for $\mathbf{c}$ TM and $\mathbf{d}$ TE polarized light extracted from $\mathbf{a}$ and $\mathbf{b}$, respectively. For both polarization states, two distinct pronounced for TE polarization and gap sizes of less than 20 $\mathrm{nm}$. In particular, this resonance peak shifts nonlinearly from $623 \mathrm{~nm}$ to $713 \mathrm{~nm}$ wavelength when the bow-tie gap size decreases from $100 \mathrm{~nm}$ to $0 \mathrm{~nm}$. This redshift of the optical resonance peak around $650 \mathrm{~nm}$ wavelength can be interpreted as coupled plasmonic interaction at the bow-tie gap. For TM polarization, the SPs are predominantly generated at the base of each triangular aperture, as shown in Fig. 1c, while for the TE polarization they are strongly localized around the nanogap, as shown in Fig. 1d. At a sufficiently small gap size, SPs induced by TE polarized light can interact and couple with each other at the nanogap, resulting in a redshift, whereas SPs generated by TM polarized light essentially do not interact because of their larger spatial separation and less dense distribution. This explanation is supported by our simulation results and the observed strong redshift for TE polarization and gap sizes below $20 \mathrm{~nm}$. The intriguing spectral shifting can be used for active optical filters, where the transmission wavelength can be controlled by the polarization of the transmitted light or by adjusting the aperture gap size.

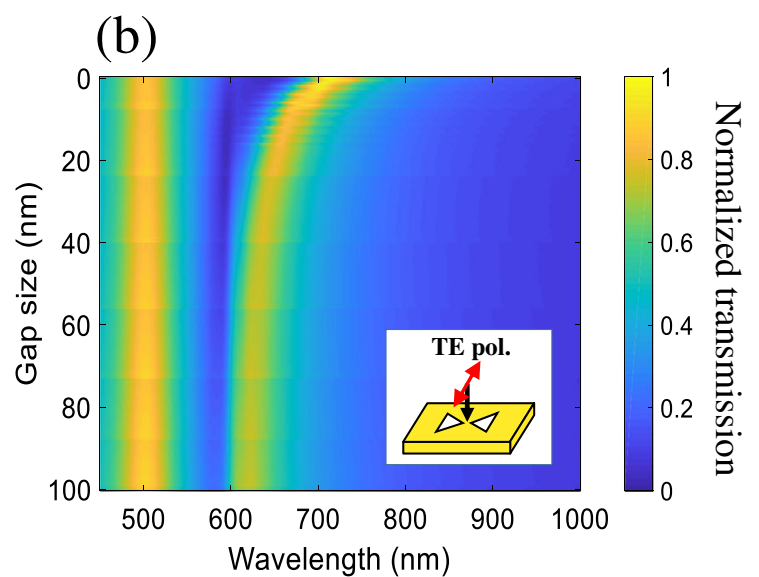

(d)

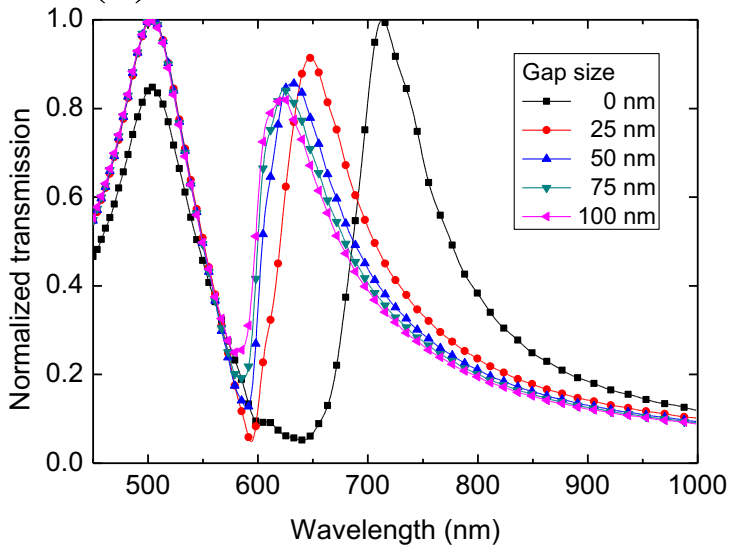

resonance peaks are identified at around $500 \mathrm{~nm}$ and $650 \mathrm{~nm}$ wavelength. While the peak position at around $650 \mathrm{~nm}$ shifts with the gap size, the position of the peak at $500 \mathrm{~nm}$ does not change for both polarization states 
The origin of EOT can be either plasmonic transmission or Rayleigh anomaly that is associated with light diffracted parallel to the grating surface. In the case of the Rayleigh anomaly, the optical transmission peak should satisfy the formula denoted in ref. [22]. The minor peak at $\sim 605 \mathrm{~nm}$ for TM polarization is not explained by the Rayleigh anomaly, based on our simulation parameters. Therefore, we expect that the peak at $\sim 605 \mathrm{~nm}$ is assumed to be one of the localized surface plasmon resonance modes whose resonance frequency is not affected by the gap of two triangular apertures.

In practice, a triangular nanoaperture with rounded corners must be considered for an experimental demonstration of EOT because of the constraints of conventional nanofabrication methods such as e-beam lithography or focused ion beam milling. Hence, we applied an effective roundness of $10 \mathrm{~nm}$ radius of curvature at each corner of the triangular apertures with all other geometrical parameters being the same as in the simulation model shown in Fig. 1b. Figures $3 a$ and b show calculated transmission spectra for ideal (no roundness) and rounded bow-tie nanoarrays, respectively, as a function of gap size between 0 and $40 \mathrm{~nm}$ for TE polarization. At a gap size of $20 \mathrm{~nm}$, the redshifted EOT peak is at $655 \mathrm{~nm}$ wavelength for the ideal bow-tie structure and $615 \mathrm{~nm}$ for the rounded bow-tie structure. Figures $3 \mathrm{~d}$ and e depict the calculated E-field distribution for TE polarization and a gap size of $20 \mathrm{~nm}$ for the ideal and rounded bow-tie structure, respectively, at their resonance wavelength. For both geometries, the induced SP significantly enhances the E-field around the nanogap; however, the enhancement factor is reduced by about $24 \%$ for the rounded bow-tie structure, because the roundness near to the nanogap reduces the plasmonic field confinement. Although the roundness reduces the overall efficiency of optical transmission through the metal film, polarization-dependent optical transmission control is still possible with practical nanostructures, which are subject to imperfections and fabrication constraints.

Figure $3 \mathrm{c}$ shows the calculated transmission as a function of gap size for a reversed bow-tie geometry using the same structural parameters as shown in Fig. $1 \mathrm{~b}$ and TE polarization. Two resonance peaks are present as in the case of ideal and rounded bow-tie geometries; however, no radical redshift of the optical transmission spectrum is observed in the reversed bow-tie geometry. Figure $3 \mathrm{f}$ shows the calculated E-field distribution at a wavelength of $634 \mathrm{~nm}$, which is the resonance wavelength for a gap size of $20 \mathrm{~nm}$ for the reversed bow-tie geometry. Although the SPs are excited at the vertex of each triangle, they do not overlap due to the spatial separation and hence cannot couple efficiently with each other, even for zero gap size. This result further supports our explanation that the drastic redshift of the transmission spectrum is due to the interaction between SPs from adjacent triangular apertures. (a)

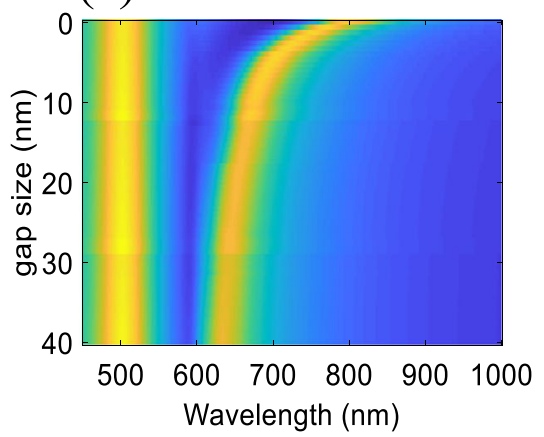

(d)

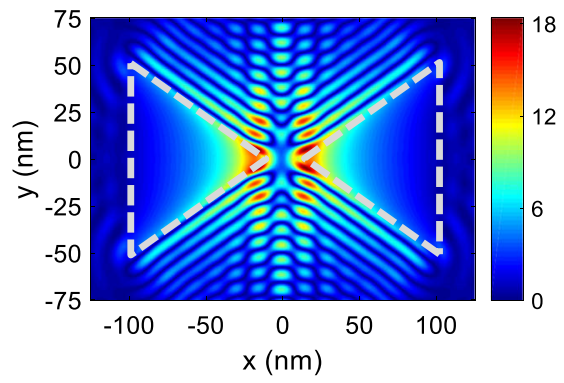

(b)

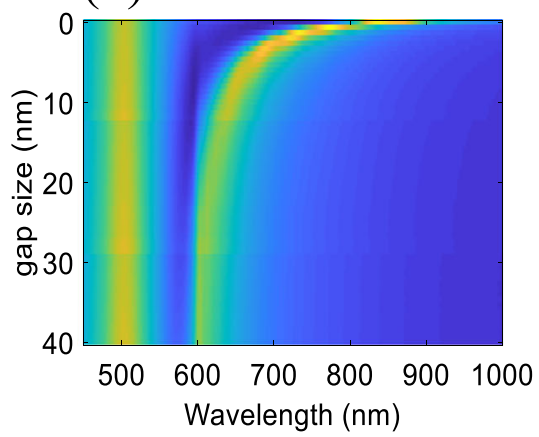

(e)

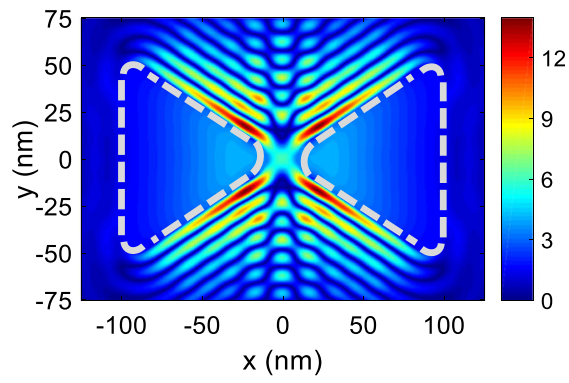

(c)

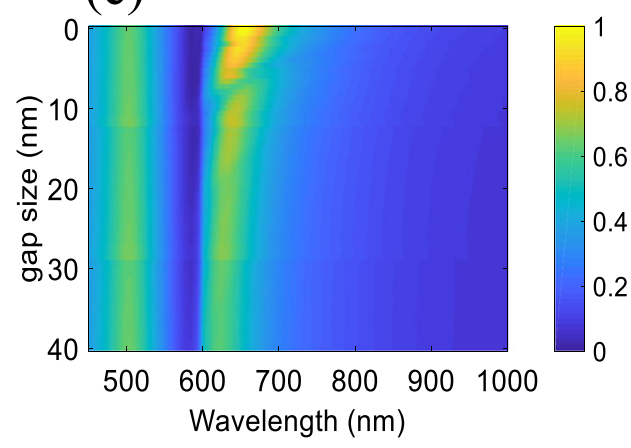

(f)

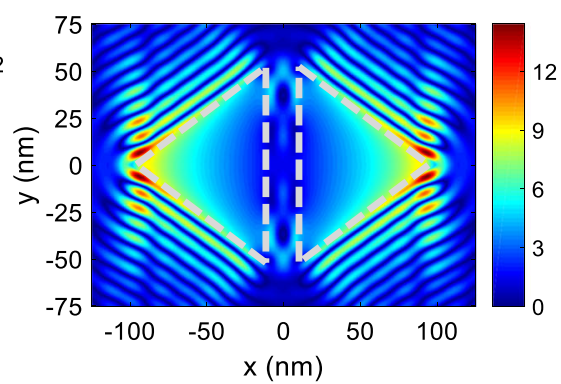

Fig. 3 Calculated transmission spectra for TE polarization as a function of gap size are shown for $\mathbf{a}$ an ideal, $\mathbf{b}$ rounded with a $10 \mathrm{~nm}$ radius of curvature, and $\mathbf{c}$ reversed bow-tie geometry. Resonant optical transmission occurs at 655,615 , and $634 \mathrm{~nm}$ wavelength for each bow-tie geometry, respectively, for a gap size of $20 \mathrm{~nm}$. d-f Calculated E-field distributions for $\mathbf{a}-\mathbf{c}$ at the corresponding resonance wavelengths for TE

polarization; the dashed lines indicate the respective bow-tie geometries. The E-field is significantly enhanced at the vertices of the triangular geometries and the strongest E-field with an enhancement factor of 18 is observed for the ideal bow-tie geometry. The rounded geometry exhibits a reduced E-field enhancement factor of 14 
Since the EOT spectrum is affected by the propagation characteristics of SPs on a metallic surface, we investigated the effect of metal film thickness on the optical redshift for TE polarization. Using the same geometrical configuration as depicted in Fig. $1 \mathrm{~b}$ and TE polarized light, we calculated optical transmission spectra for different film thicknesses of 50,100,150, 200, and $300 \mathrm{~nm}$, which are shown in Fig. 4b-f, respectively. An optical redshift was observed for all cases when varying the bow-tie gap from 0 to $20 \mathrm{~nm}$. Notably, a lower optical transmission is observed for increasing metal film thickness because of intrinsic plasmonic losses in metal. The position of the resonance peak around $650 \mathrm{~nm}$ wavelength slightly shifts with a change of film thickness because it affects the plasmonic resonance condition. For film thicknesses of $50 \mathrm{~nm}$ and $100 \mathrm{~nm}$ (Fig. 4b, c), the redshifting EOT resonance is a well-defined single peak. For thicker films, however, the EOT peak becomes irregular, and a multi-peak structure appears for small gap sizes (Fig. 4d-f). This irregularity of the EOT peak for thicker films may be due to the additional excitation of resonant out-of-plane plasmonic modes along the depth of the nanoaperture [22-24]. Nevertheless, the redshift clearly appears over a large range of film thicknesses, which is useful for the design of active optical filters, considering practical limitations of typical nanofabrication methods. Limiting the film thickness additionally improves performance by providing more consistent transmission characteristics.

\section{Conclusion}

In summary, we showed that the optical transmission spectrum by plasmonic EOT could be redshifted by a variation of the bow-tie nanogap rather than by changing the pitch of the array. Intriguingly, the drastic shift of the optical transmission peak for gap sizes below $20 \mathrm{~nm}$ is only observed for TE polarized light. This is due to the strong plasmonic coupling between SPs excited at the vertices of adjacent triangular structures by the TE polarized light. In practice, the redshift can be controlled by the polarization state of the input light beam or by changing the aperture gap size on a nanometer scale. Therefore, this induced spectral shift of the extraordinary optical transmission can be utilized for various applications such as active optical filters or displays.

Author Contributions Statement The project was planned and overseen by S.K., K.K., and J. P. The simulations are performed by J.P and H. L. Data were analyzed by S.K. J.P and A.G. All authors contributed to the discussion and preparation of the manuscript.

Funding Information This work was supported by the National Research Foundation of the Republic of Korea (NRF-2017R1C1B2006137), ICT, Future Planning (NRF-2017M3D1A1039287), and Basic Research Lab Program (NRF-2018R1A4A1025623). (a)

Top view

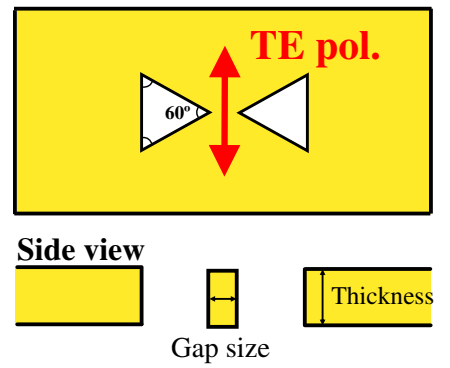

(d)

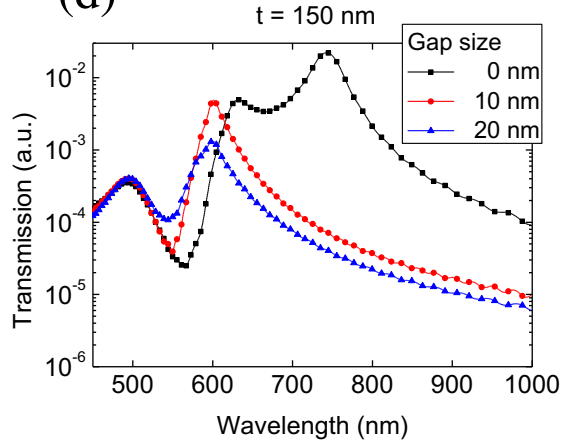

(b)

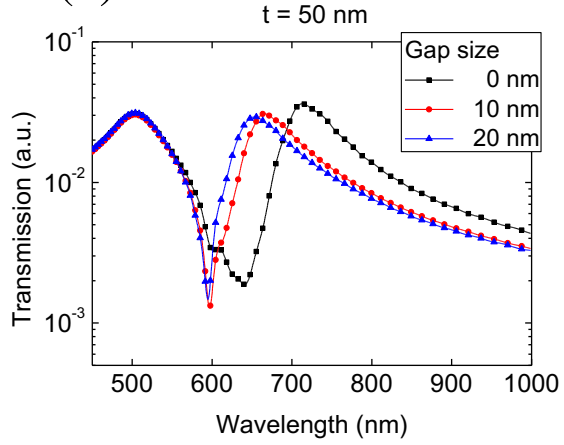

(e)

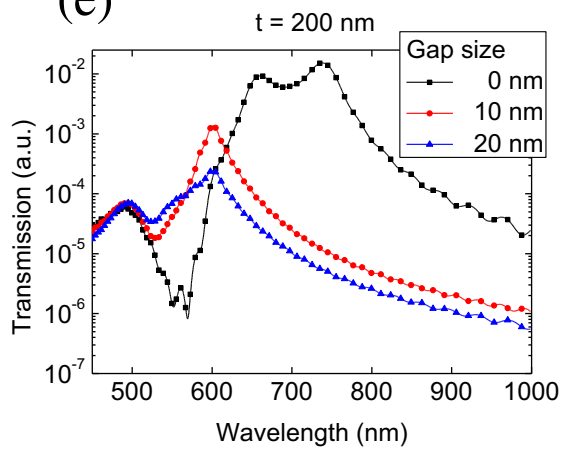

(c)

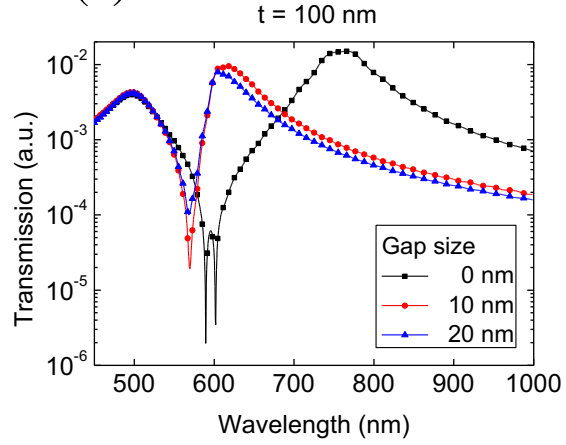

(f)

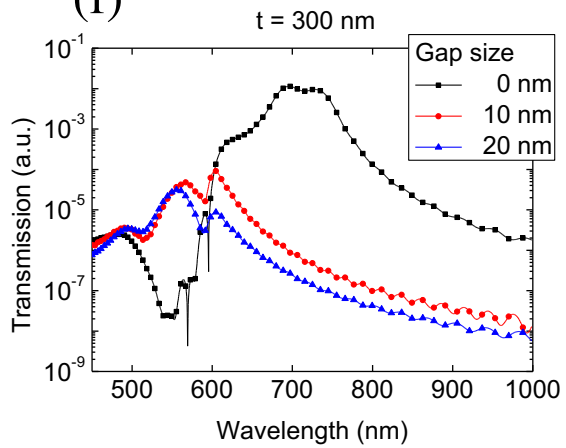

Fig. 4 Transmission spectra for TE polarized light in dependence on film thickness. a Geometry of the bow-tie structure. $\mathbf{b}-\mathbf{f}$ Transmission spectra for a film thickness of 50,100, 150, 200, and $300 \mathrm{~nm}$, respectively. For each thickness value, transmission spectra were calculated for gap sizes of
0,10 , and $20 \mathrm{~nm}$. The redshifted EOT peak becomes irregular for film thicknesses above $100 \mathrm{~nm}$ and exhibits a multi-peak structure for small gap sizes 
Open Access This article is distributed under the terms of the Creative Commons Attribution 4.0 International License (http:// creativecommons.org/licenses/by/4.0/), which permits unrestricted use, distribution, and reproduction in any medium, provided you give appropriate credit to the original author(s) and the source, provide a link to the Creative Commons license, and indicate if changes were made.

\section{References}

1. Ebbesen TW, Lezec HJ, Ghaemi HF, Thio T, Wolff PA (1998) Nature 391:667

2. Garcia-Vidal FJ, Martin-Moreno L, Ebbesen TW, Kuipers L (2010) Rev Mod Phys 82:729

3. Mark I (2011) Stockman. Opt Express 19(22):22029

4. Kauranen M, Zayats AV (2012) Nat Photonics 6:737

5. Fischer H, Martin OJF (2008) Opt Exp 16(12):9144

6. Klein Koerkamp KJ, Enoch S, Segerink FB, van Hulst NF, Kuipers L (2004) Phys Rev Lett 92:183901

7. Degiron A, Ebbesen TW(2005) J Opt A Pure Appl Opt 7(2)

8. Kim S, Jin J, Kim Y-J, Park I-Y, Kim Y, Kim S-W (2008) Nature 453:757

9. Willets KA, Van Duyne RP (2007) Rev Phys Chem 58:267

10. Wurtz GA, Zayats AV (2008) Laser Photonics Rev. 2(3):125

11. Wang L, Uppuluri SM, Jin EX, Xu X (2006) Nano Lett 6(3):361

12. Barnes WL, Dereux A, Ebbesen TW (2003) Nature 424:824

13. Anh ND, Chun BJ, Choi S, Kim D-E, Kim S, Kim Y-J (2019) Nat Phys 15:132
14. Ishiharaa K, Ohashi K (2006) Appl Phys Lett 89:201120

15. Tame MS, McEnery KR, Özdemir ȘK, Lee J, Maier SA, Kim MS (2013) Nat Phys 9:329

16. Melikyan A, Alloatti L, Muslija A, Hillerkuss D, Schindler PC, Li J, Palmer R, Korn D, Muehlbrandt S, Van Thourhout D, Chen B, Dinu R, Sommer M, Koos C, Kohl M, Freude W, Leuthold J (2014) Nat Photonics 8:229

17. Haffner C, Heni W, Fedoryshyn Y, Niegemann J, Melikyan A, Elder DL, Baeuerle B, Salamin Y, Josten A, Koch U, Hoessbacher C, Ducry F, Juchli L, Emboras A, Hillerkuss D, Kohl M, Dalton LR, Hafner C, Leuthold J (2015) Nat Photonics 9:525

18. Dennis BS, Haftel MI, Czaplewski DA, Lopez D, Blumberg G, Aksyuk VA (2015) Nat Photonics 9:267

19. Kim S, Jang MS, Brar VW, Tolstova Y, Mauser KW, Atwater HA (2016) Nat Commun 7:12323

20. Jin EX, Xu X (2006) Appl Phys Lett 88:153110

21. Yakubovsky DI, Arsenin AV, Stebunov YV, Fedyanin DY, Volkov VS (2017) Opt Exp 25(21):25574

22. Gao H, McMahon JM, Lee MH, Henzie J, Gray SK, Schatz GC, Odom TW (2009) Rayleigh anomaly-surface plasmon polariton resonances in palladium and gold subwavelength hole arrays, 2340. Opt Express 17(4):2334

23. Anishur Rahman ATM, Majewski P, Vasilev K (2012) Opt Lett 37(10):1742

24. Liu F, Zhang X (2015) Biosens Bioelectron 68:719

Publisher's Note Springer Nature remains neutral with regard to jurisdictional claims in published maps and institutional affiliations. 